\title{
The effects of Dohsa-hou relaxation skill training on indicators of mood state regulation in Japanese junior high school students
}

Yu Kawamata, Takuya Fujikawa, Yuka Mizuno, Xinyi Liu, Yutaka Haramaki

Graduate School of Education, Hiroshima University, Higashihiroshima, Hiroshima, Japan

\section{Background}

This study aimed to offer relaxation skills using Dohsa-hou to junior high school

students and investigate the effect of Dohsahou on their mood states. This study was conducted in response to a principal's request to facilitate ways to regulate behavior and allow students to concentrate on learning.

\section{Dohsa-hou}

Dohsa-hou is an original Japanese psychotherapy that focuses on stabilizing the body as a means to change the mind by relaxing physical strain and controlling the self. The theory of Dohsa-hou is framed along a general process of intention, effort, and the realization of an intended body movement that conforms to the subjective experience of motor resonance. A distinction about the self rather than another as the agent of control and movement performance change is proposed in Dohsa-hou, and empirical support from clinical case studies in Japan has indicated its psychotherapeutic utility for autism, schizophrenia, and others.

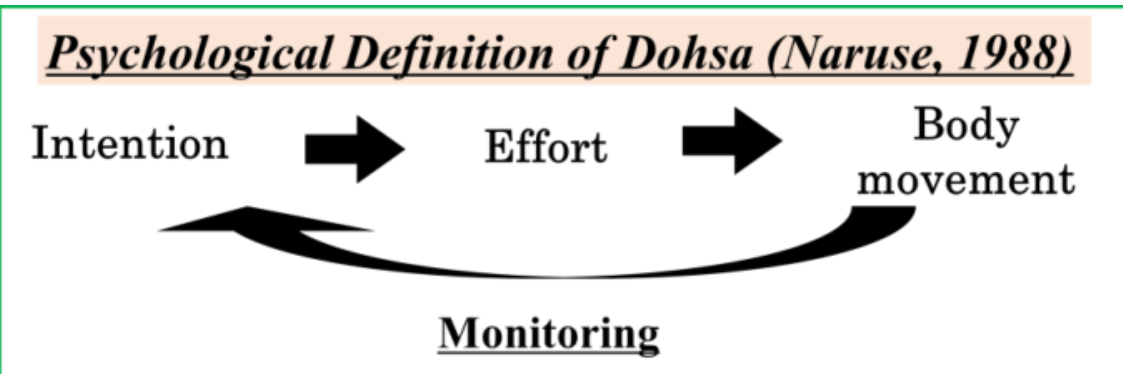

\section{Method}

Participants: 41 Japanese junior high school students at Hiroshima (23 males, 18 females, $M$ age $=13.7)$

\begin{tabular}{|c|l|}
\hline Procedure & \multicolumn{1}{|c|}{ Detail } \\
\hline (1)Pre-POMS2-Y & $\begin{array}{l}\text { Respond to a 5-point Likert scale based on } \\
\text { hou they were feeling the day. }\end{array}$ \\
\hline (2Relaxation task & $\begin{array}{l}\text { Raise and lower their shoulder by } \\
\text { themselves and in pairs. }\end{array}$ \\
\hline 3)Post-POMS2-Y & Same as the pre-POMS2-Y \\
\hline
\end{tabular}

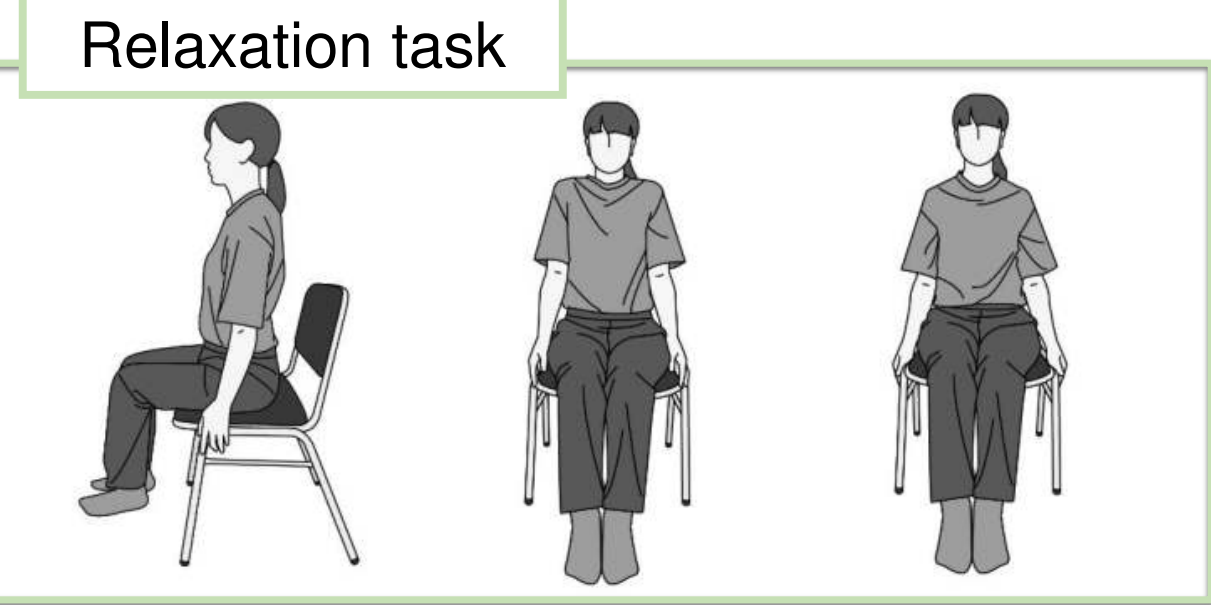

The POMS2-Y simultaneously measures six mood states (Depression-Dejection, Vigor, Anger-Hostility, Fatigue, Tension-Anxiety, and Confusion).

\section{Findings}

(1) we conducted the item analysis for each factor of POMS2-Y, and acceptable reliability coefficients were shown for all factors: Depression-Dejection ( $\alpha=.840$ ), Vigor $(\alpha=.822)$, Anger-Hostility ( $\alpha=.829)$, Fatigue $(\alpha=.841)$, Tension-Anxiety (.880), and Confusion $(\alpha=.801)$.

(2)as a result of paired sample t-tests (Figure 1), the mood states scores excluding Vigor decreased. The effect size was especially larger in Fatigue $(t(77)=5.324, p<0.01$ Cohen's $d=0.514)$, Tension-Anxiety $(t$ $(77)=6.477, p<0.01$; Cohen's $d=0.683)$, and Confusion $(t(77)=5.656, p<0.01$; Cohen's $d=0.574)$ ". This decrease was also observed for Total Mood Disturbance (TMD) ( $t$ (77) $=5.948, p<0.01$; Cohen's $d=0.559$ )

(1) and (2) results show that mood states related to negative feelings decreased at post-test as a result of the relaxation experience using Dohsa-hou and indicated an overall change to a more stable mood state.

\section{Discussion}

This study showed that Dohsa-hou demonstrated specific effects for lowering negative mood states and stabilizing TMD in a non-clinical sample of junior high school students. A notable point is that the score of the Vigor did not decrease. This indicated that Dohsa-hou might only decrease the negative mood states without lowering the positive mood state in junior high school student stress management settings.

In general, children are thought to experience stress from schoolwork and interpersonal relationships in junior high school, as it is a time when various problems such as school refusal and bullying are known to occur. This application of Dohsa-hou has shown some capacity to contribute to such efforts by providing a skill to improve the mood states of children who may encounter these issues or benefit from behavioral self-regulation.

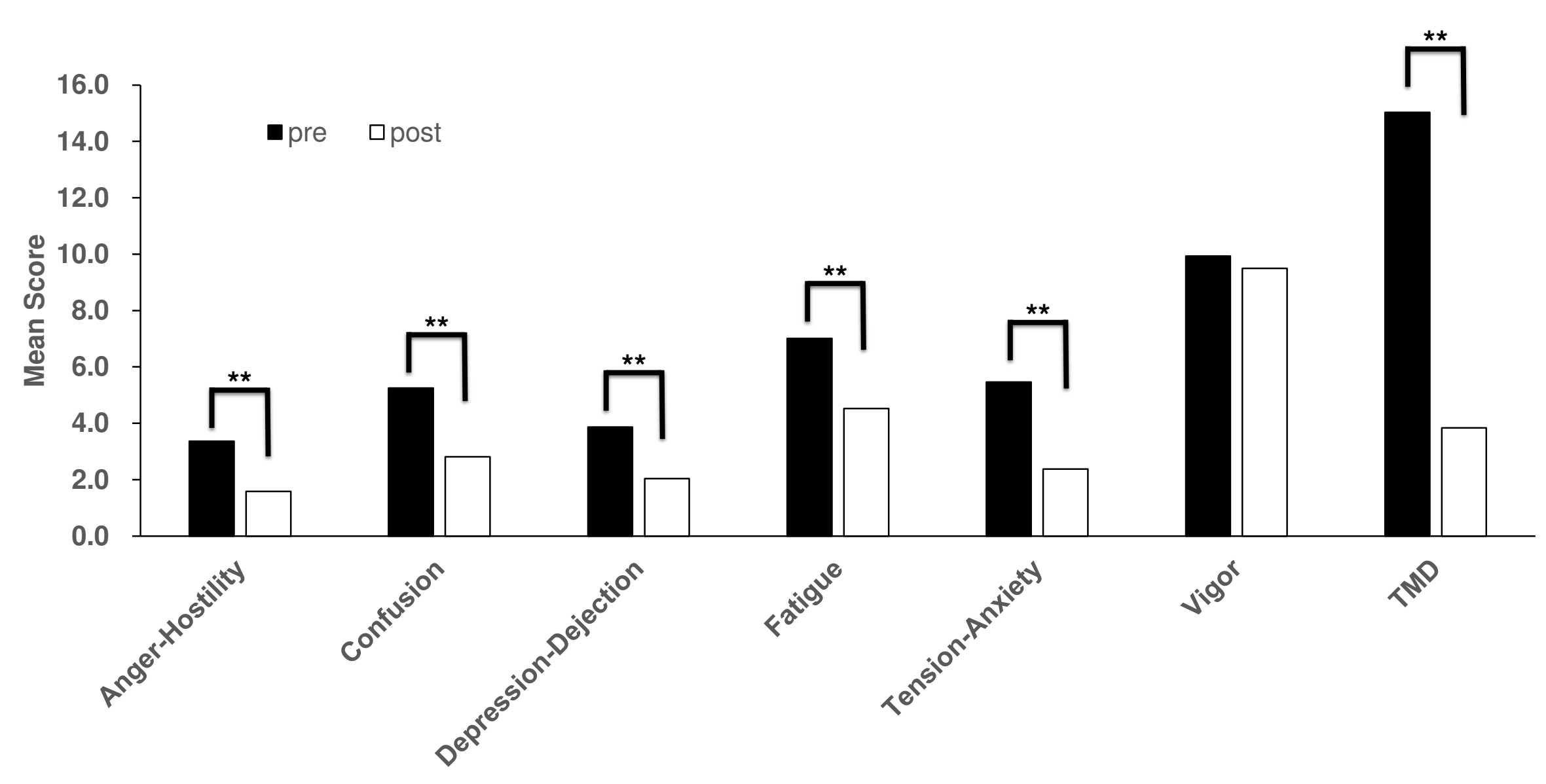

Figure 1. Paired sample $t$-tests

\section{Limitations}

This study was a pre-post test of mean value differences in mood states from a relaxation skill training workshop that lacked a control group for comparison. Future studies should compare these effect sizes with other conditions, as well as possibly account for trait level influences on the degrees of change from participation in the intervention. 